Short communication

\title{
A potato cDNA encoding a homologue of mammalian multidrug resistant P-glycoprotein
}

\author{
W. Wang, D. Takezawa and B.W. Poovaiah* \\ Laboratory of Plant Molecular Biology and Physiology, Department of Horticulture, Washington State University, \\ Pullman, WA 99164-6414, USA (*author for correspondence) \\ Received 26 September 1995; accepted in revised form 13 February 1996
}

Key words: calmodulin, multidrug resistance, P-glycoprotein, tuberization

\begin{abstract}
A homologue of the multidrug resistance (MDR) gene was obtained while screening a potato stolon tip cDNA expression library with ${ }^{35} \mathrm{~S}$-labeled calmodulin. The mammalian MDR gene codes for a membrane-bound $\mathrm{P}$ glycoprotein $(170-180 \mathrm{kDa})$ which imparts multidrug resistance to cancerous cells. The potato cDNA (PMDR1) codes for a polypeptide of 1313 amino acid residues (ca. $144 \mathrm{kDa}$ ) and its structural features are very similar to the MDR P-glycoprotein. The N-terminal half of the PMDR1-encoded protein shares striking homology with its $\mathrm{C}$-terminal half, and each half contains a conserved ATP-binding site and six putative transmembrane domains. Southern blot analysis indicated that potato has one or two MDR-like genes. PMDRI mRNA is constitutively expressed in all organs studied with higher expression in the stem and stolon tip. The PMDR 1 expression was highest during tuber initiation and decreased during tuber development.
\end{abstract}

Multidrug resistance (MDR) is a phenomenon known to occur in mammalian tumor cells that hampers cancer chemotherapy $[5,6,14]$. The MDR gene codes for a membrane-bound $\mathrm{P}$-glycoprotein that functions as an ATP-dependent efflux pump which extrudes a range of structurally different compounds from the cell, including cytotoxic natural products [6]. Multidrug-resistant cells are characterized by increased expression of the MDR gene and decreased intracellular drug accumulation. The MDR P-glycoprotein belongs to the ATPbinding cassette $(\mathrm{ABC})$ superfamily of membranebound transport proteins [7]. A number of MDR-like genes have been cloned from both eukaryotes and prokaryotes [6, 14]. Eukaryotic P-glycoproteins are characterized by the presence of 12 transmembrane domains within the two similar halves, each half containing six transmembrane regions and an ATP-binding

The nucleotide sequence data reported will appear in the EMBL, GenBank and DDBJ Nucleotide Sequence Databases under the accession number 452079 site within the large intracytoplasmic loop. An Arabidopsis genomic sequence with homology to mammalian MDR-like genes has been reported [4].

A ${ }^{35} \mathrm{~S}$-labeled potato calmodulin isoform PCM6 [16] was used for screening a potato stolon tip cDNA expression library ( $\lambda$ ZAPII, Stratagene) to obtain calmodulin-binding clones [11]. After three rounds of screening, several positive clones were identified. Among these clones, a partial cDNA clone was sequenced (3172 bp, nt 953-4124, Fig. 1) and the sequence comparison using the GenBank database revealed that it had high homology to MDR-like genes. In order to clone the $5^{\prime}$ region, the cDNA library was rescreened using a ${ }^{32} \mathrm{P}$-labeled EcoRI/SpeI fragment (nt 953-1710, Fig. 1) of the original clone as a probe. Out of five positive clones obtained, two cDNA clones (nt $1-3548$, Fig. 1) contained longer ${ }^{\prime}{ }^{\prime}$ regions and another three cDNA clones (nt 321-4160, Fig. 1) contained 26 additional nucleotides in the $3^{\prime}$ region as compared to the original cDNA. Since the most 
1 TANAAACCATTGGACATTGGAAATGGTCTGAA

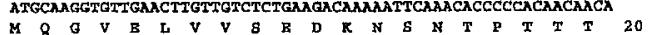
93 ACAACAACAANTAGTCATCAATTTCAMGAACAAGAATGGAGGTTAגANAAGAAGAAGG

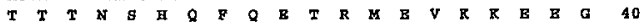

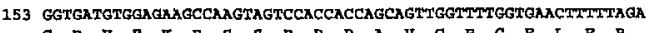

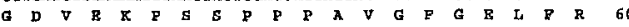
213 TTTGCTGATGGTTGGATTGTGTACTAATAATKRTTGGTTCACTTGTGCTTPTGTCCAT F A D G L D C V L $M$ I I G S

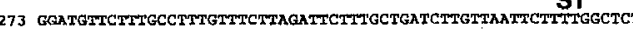
G C S L D L P L R F F A D L W N S F G S 100 333 TATGCTAATGATGTTGATAAGATGACTCAAGAGTTTTAAAGSATGCATTTIACTTTCTT $Y$ A N D V D R M T Q

393 GTGGTGGGTGCTGCAATATGGGCATCXTCATGGGCAGXGATATCATGCTGGATGTGGACT

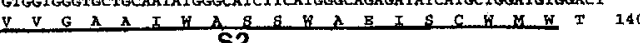
453 GGTGAGXGACAAXCAACAAAGATGAGGATCAARTACTTAGAGGCTGCTTPGAACCAAGAT G E R O I T X M R I K Y L B A A L N Q D 160 513 ATTCAATATTTTGATACTGAAGTMAGACTTCTGATGTTGTTTCTGCAATTAaCACTGAT

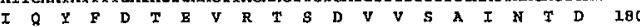

573 GCTGTAGTGGTCCAXGATGCCATTAGTGAGAAGTTGGGCAA7TTCATTCATTATATGGCT

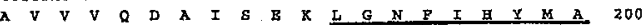

633 ACATTYTTGTCTGGATTTGTGGTGGGATTTACAGCAGTATGGCAATTAGCTCTAGTTACT

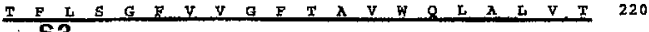
S3

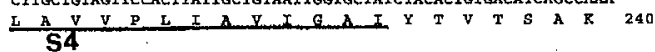
TH

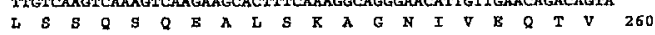
日13 GTTCAMATTCGGACGeTATTGGTGTTTGTTGGTCAGGCAAAAGCATTGCAAGCATACACA

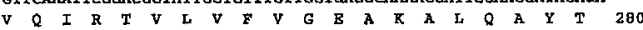

873 GCAGCACTTAGAGTTTCTCAAAAGATTGGATATAAGAGTGGATTMTCAAAAGGATTAGGA

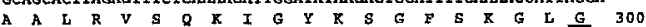

933 CITGGAGCTACATATTTTACTCXTTTCTGTTGTTATGCTCXTCTITTATGGTATGGGGG

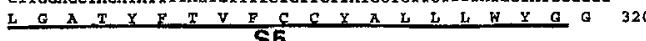

993 TATTTAGTTAGACATCATTTCACCAATGGAGGACTTGCCATAGCAACAATGTTTGCAGTC $X$ I $\quad V \quad R$ H 1053 ATGATTGGTGGATTGGCATTGGGACAATCTOCCCCTAGCATGACTGCATZTLCAAAMGCI B I G G L A I $G$ G 1113 AGAGTTGCAGCTGCCAAGATTTTCCGGATTATCGATCATAAGCCAAGCGTCGACAGAAAC

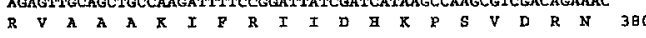

1173 GCCAAGACGGGGTTGGAGTTAGACACTGTTAGTGGCCAGCTAGAGCTTHAGAATETCGAG A K T G L E L L D T V S G Q L L E L E K N V

1233 TTCTCTTATCCITCAAGGCCTGAAATCAAGATTCTCAACAATTTCAACCTCGTTGTTCCA F S Y P B P E I K I L N F N L V V P 120

1293 GCTGGAAAGACCATCGCTTTAGTCGGAAGCAGTGGTTCGGGGAAAAGCACTGTGGTATCC

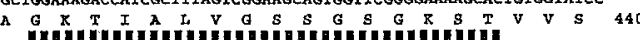

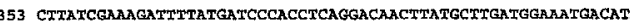

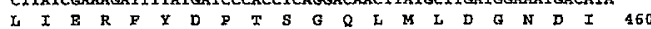

1413 AAGACACTGAAATTGAAATGGCTAAGGCAGCAAATTGGCCTIIGTAAGCCAAGAACCAGCA $\begin{array}{lllllllllllllllllllll}\mathbb{K} & \text { T } & \text { I } & \mathrm{K} & \text { L } & \mathrm{K} & \text { W } & \text { L } & \text { R } & Q & \text { Q } & \text { I } & \text { G } & \text { L } & \text { V } & \text { S } & \text { Q } & \text { E } & \text { P } & \text { A } & 480\end{array}$ 1473 CTTITCGCAACAAGCATCAAAGAAAACATACTATRAGGAAGGCCACATGCAACACAAATT $\begin{array}{lllllllllllllllllllll} & F & A & T & S & I & K & E & N & I & E & L & G & R & P & D & A & T & Q & I & 500\end{array}$

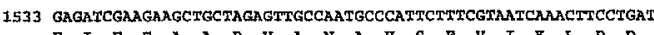

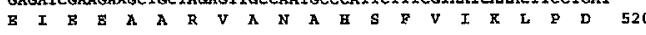

1593 GGCTTTEATACTCAGGTAGGGGAGAGAGGATTACAATTATLAGGTGGACAGAAGCAGAGG

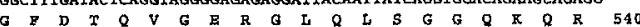

1653 ATTGCTATAGCAAGGGCCATGCTTAAGAATCCAGCGATCCTTCTCTTAGACGAGGCAACT

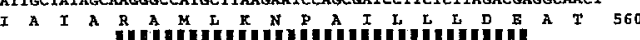

713 AGTGCTTTAGATTCCGAATCAGAAAAGCTAGTGCAGGAGGCTCTAGACAGGTTCATGATC

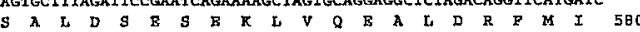

1773 GEXCGAACGACTCTTGIGATTGCTCATCGGCTGTCTACTATCCGCAAGGCTGACCIGGTG

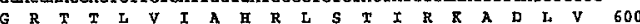

1833 GCTGTACTACAACAAGGCAGTGTCXCGGAGATTGGAAGCCACGATGAGCTPATOAGTAAX

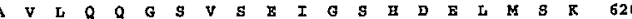

1993 GGAGAGAATGGTATGTATGCCANGCTCATCAAAATGCAAGAAGCAGCTCATGAAACCGCT

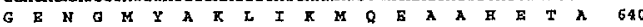
2953 CTTAGTARTGCCAGAhAGAGCAGTGCAMGGCCCTCGAGTGCARGGAACTCTGTAAGCTCX

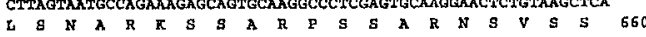
2013 CCANTCATCACTAGAMACTCTTCCTATGGTCGATCACCATACTCCCGCCGGTTGTCTGAC P I I T R N S S Y G R S P Y S R R L B D 690
2073 TITTCSACCTCGGACTICAGCCTCYCCCHTGATGCTGCATATHCTAATHACCGAMATGAA F S I S 5 S 133 AגGCTTGCATTCAAGGACCAAGCTAGTTCGRYTGGCCGGCTTGCAAAAATGAACTCTCCO $\begin{array}{lllllllllllllllllllll}\text { C } & \text { L } & \text { A } & \text { F } & \text { K } & \text { D } & \text { Q } & \text { A } & \text { S } & \text { S } & \text { P } & \text { G } & \text { R } & \text { L } & \text { A } & \text { K } & \text { M } & \text { N } & \text { S } & \text { P } & 720\end{array}$

2193 GAGTGGACTTATGCTTTAATTGSTTCTATAGGCTCTGTCATCTGTGGTTCACTTAGTGC I W T Y A L I G S I G S V I C G S L L S A 740 253 TTCTTFGCATACGTCTTEAGTGCTGTTCTTAGCGTGTACTACAATCCGGATCATGCTIA

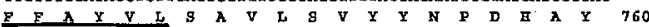

2313 ATGAGCGAACAAATTGCGAAMTACTGTTATCTIITIGATIGGAGTTTCATCGGCTGCACTC M S B 2373 ATT2TCAACACTCTACAGCATTACTACTGGGATGTAGTGGGGGAGAATTTAACAAAACGG I F N T L Q E Y Y W D V V G B N L T R R 000 233 GTGAGAGAGAAATECTTGOCAGCAGTGCTTAAAMTGGAAATGGCGTGGTTCGATCAGGAR V R E K M I A A V L K M E M A W F D Q E 820 2493 GAGAACGATAGTTCAAGANTTGCAGCTAGGCTCTCTCTGGATGCCAACAATGTTAGGTCA

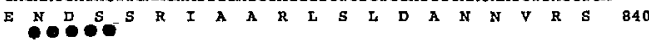
2553 GCCAMTGGGATAGAATCTCCGTCATTATGCAGAACTCAGCTCTCATGCTAGTCGCOTGC A. I G $D$ R I S Y I \& Q N S A I M I V A C 860 213 ACTGCAGGATTCGTATKGCAGTGGCGTCTGGCCCTCGXCTCATTGGGGTCTTCCCCGT I A G F V I 8 H 2673 GTCGTTGCAGCAACAGTTTRACAGAAAATGTTCATGAAGGGATTCTCAGGAGACTTGEAA

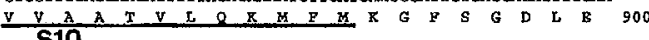
S10
GCTGCTCATGCCAAAGCCACTCAACTTGCTGGAGAAGCTGTAGCTAATGTAAGAACAGTM

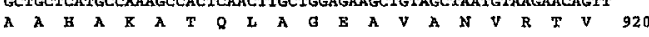

2793 GCTGCCTTIAATTCGGAGACGAAAATAGTCAATCTITTCGACTCCAGCCTCCAAACTCCG

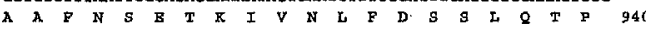

2853 CTTAGGCGTTGCTTCTGGAAGGGACAGATTGCGGGAAGTGGTTATGGGATTGCTCAATTT I R R C F W R G Q I A G S G Y G I A Q E 960

2913 TTGCIITATTCTTCCTATGCCCTTGGCCTITGGTATGCCTCCTGGCTTGTCAAGCATGG L L Y S S X A L G L H Y A S W I V X E G 980 973 ATCTCTGACTTCTCGAAGACGATCCGTGTTITCATGGTGCTCATGGTTTCTGCTMATGOI

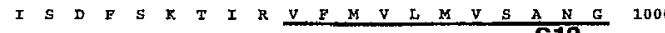

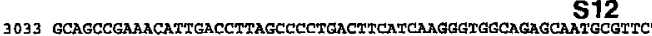
$A$ A E I I I L A P D F I R G G R A M R S 1020 3093 GTTTPCGACTCCTTEACCGTAAAACAGAAGTTGAGCCGGATGATCCAGATGCCACCOCT

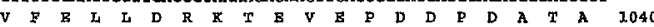
3153 GTCCCTEATCGTCITCGTGGTGAGTGGATTTAAGCATGLAGACTTCTCATATCCCACT

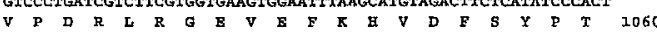
3213 AGACCCGACGTGTCAATTTTCCGTGATTTGAATCTTCGTGCTCEAGCTGEAAAOACTCTT

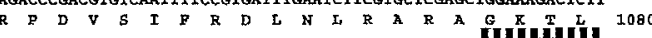
3273 GCTCTTGTTGGACCAAGTGGATGTGGKAAGAGCTCAGTCATTICACMTATAGAGCGGTTC

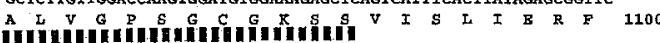
333 TACGAGCCATCATCTGGACGTGTCATCATCGATGGCAAGGATATTCGTAAGTACMACET

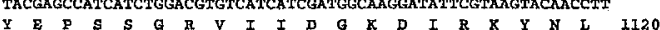
3393 AAATCCTTGAGAAGACACATTGCTGTAGTGCCACAAGAXCCTTGCCTCTTTGCTACCAC $\begin{array}{lllllllllllllllllllll}\text { R } & \text { S } & \text { L } & \text { R } & \text { R } & \text { A } & \text { I } & \text { A } & \text { V } & \text { V } & \text { P } & \text { Q } & \text { B } & \text { P } & \text { C } & \text { L } & \text { F } & \text { A } & \text { T } & \text { T } & 1140\end{array}$ 3453 ATCTATGAAAACATCGCGTATGGACATEAATCAGCAACCGAGGCTGAGATAACCGARGCX

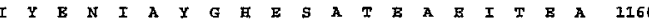

3513 GCAACEXGGCAAACGCCCACAAGTTCATATCTECATTGCCTGATGGATACAAAACATTT

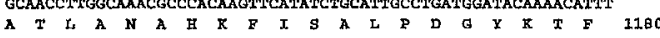
3573 GTTGGAGAAAGGGGAGTTCAATTGTCTGGTGGACAAAAGChAAGAATCGCCATTGCTCGT

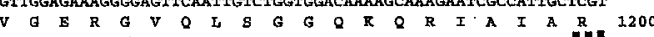

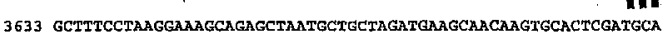

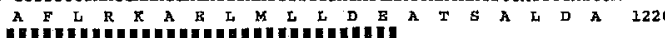
693 GAGTCTGAAAGATGTGTACAAGAAGCATTGGATCGCGCTTGTGCTGGTAAGACCACTAT

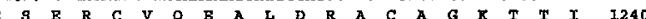
3753 GTTGTTGCACACAGGCTATCTACAATCAGAAATGCACATGTGATCGCGGTCATAGACGA

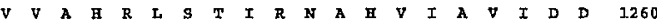
3013 GGGAAGGTGGEGAACARGGTTCTCATTCTCATCTGTTGAAAAACTACTCAGATGGTAT G X V A E $Q$ G G S 3873 TATGCGCGTATGATACAACTACAACGATTTACACACGGAGAAGCTGTGAATATGGCAACA

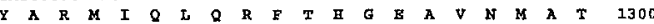
3933 GGATCAACGTCTTCCTCGCGTCCTAAGGAAGATCAAGATTGAGAAGAAAACGCGAATFGA

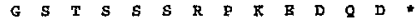

3993 AgCTCAAMATGhaAaAATACCAGTAGAaAMaAaAGGGMGTATACCTTGTGTAACACCCTT 4053 ATTATTXTTTGGACTGCTAATYYCTTGTTCACTGTTTCRAGTATAGTATAGTATAATAT

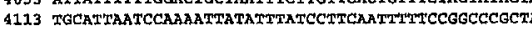

Figure 1. Nucleotide and deduced amino acid sequences of the PMDRI gene. Putative transmembrane segments S1-S12 are underlined. Two potential ATP-binding sites (each consisting of two parts) are indicated by hatched regions. The dotted lines indicate the potential N-linked glycosylation sites corresponding to the sewaences NXS/T. The HindIII site is indicated by a double line above the sequence. 

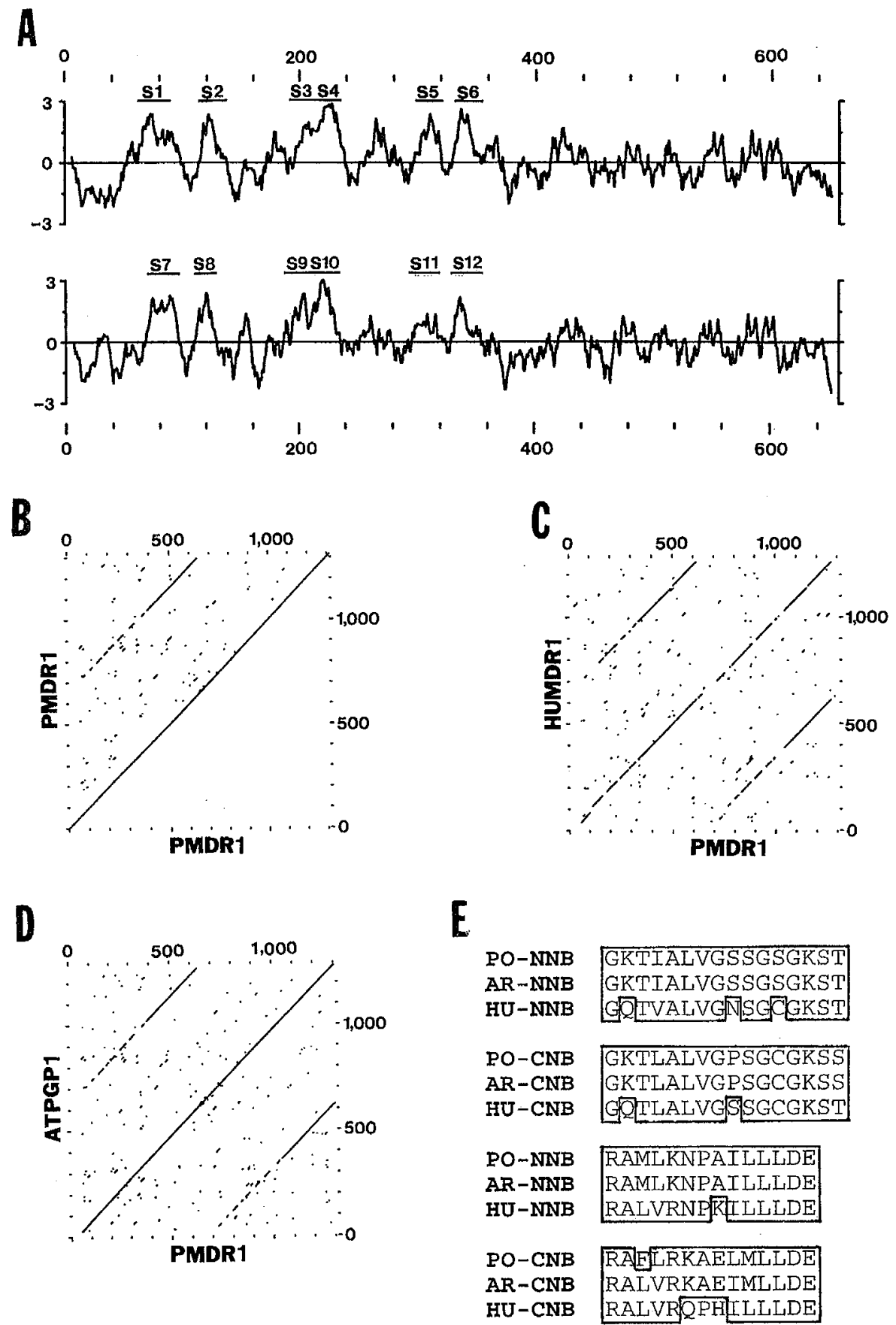

Figure 2. A. Hydropathy plot of the predicted amino acid sequence of the N-terminal half (top) and C-terminal half (bottom) of PMDR1. Kyte-Doolittle hydrophobicity values are marked on the left for a window of 11 amino acid residues. Putative transmembrane segments of $\mathrm{S} 1-\mathrm{S} 12$ are indicated. B. Dot matrix comparison showing internal duplication in the PMDR1 polypeptide. C. Dot matrix comparison of human MDRI P-glycoprotein (HUMDR1) to the potato MDR-like gene product (PMDR1). Dot matrix analysis was performed for a window of 19 amino acid residues. D. Dot matrix comparison of Arabidopsis P-glycoprotein (ATPGP1) to PMDR1. E. Comparison of the potential ATP-binding sites of PMDR1 to those of Arabidopsis ATPGP1 and human MDR1 genes. Identical and functionally similar amino acid residues are boxed. PO, AR and HU indicate potato, Arabidopsis, and human, respectively. NNB and CNB represent the conserved sequences in the ATP-binding sites of the $\mathrm{N}$-terminal half and C-terminal half, respectively. 
upstream ATG at position 23 is followed by a termination codon after only three nucleotides, we assign the ATG at nucleotide position 33 as the translation initiation codon, which is located 2 bases downstream from a purine residue in agreement with the eukaryotic initiation site [9]. The $186 \mathrm{bp}$ of the $3^{\prime}$-untranslated region sequence does not contain an intact polyadenylation signal and an obvious poly $(\mathrm{dA})$ tail and they may be present in the downstream region. The cDNA codes for a polypeptide of 1313 amino acid residues. The complete nucleotide sequence and the deduced amino acid sequence of PMDR1 are presented in Fig. 1.

The hydropathy profiles and the dot matrix comparison revealed that the $\mathrm{N}$-terminal half of PMDR 1 is similar to its C-terminal half (Fig. 2A, 2B), and their amino acid sequences share $36.8 \%$ identity and $59.8 \%$ similarity. The dot matrix comparison (Fig. 2C) between PMDR1 protein and human MDR1 P-glycoprotein [3] shows that their amino acid sequences share extensive homology $(41.1 \%$ identity and $63.7 \%$ similarity). Fig. 2D shows the high homology between PMDR1 and the gene product of ATPGP1, a genomic clone which was isolated from Arabidopsis [4]. Their amino acid sequences share $85.7 \%$ identity and $92.4 \%$ similarity. However, a distinct variation exists in their N-terminal ends; the PMDR1 polypeptide has an extra 26 amino acid stretch in the $\mathrm{N}$ terminal end as compared to Arabidobsis ATPGP1encoded protein. The deduced amino acid sequence of PMDR1 revealed several significant structural features. The hydropathy plot presented in Fig. 2A shows a series of highly hydrophobic domains in the $\mathrm{N}$-terminal and C-terminal halves and their hydropathy profiles are very similar (Fig. 2A). Each half of the PMDR1 polypeptide contains six putative transmembrane segments (Fig. 1, 2A) which are conserved in the corresponding regions of other MDR homologues. Another structural feature of PMDR1 polypeptide is the presence of two putative ATP-binding sites (Fig. 1). Two pairs of conserved amino acid sequences are present in the polypeptide at positions $422-437 / 545-558$ in the $\mathrm{N}$-terminal half and positions $1077-1092 / 1200-1213$ in the C-terminal half. These amino acid sequences of the putative ATP-binding sites are highly conserved in proteins coded by MDR-like genes (Fig. 2E).

To determine the approximate copy number of PMDR1, Southern blot analysis of potato genomic DNA was carried out using the random-primed ${ }^{32} \mathrm{P}-$ labeled probe of the cDNA fragment (nt 953-3302, Fig. 1) [13]. Depending on the restriction enzymes, one or three hybridizing bands were observed, indi-
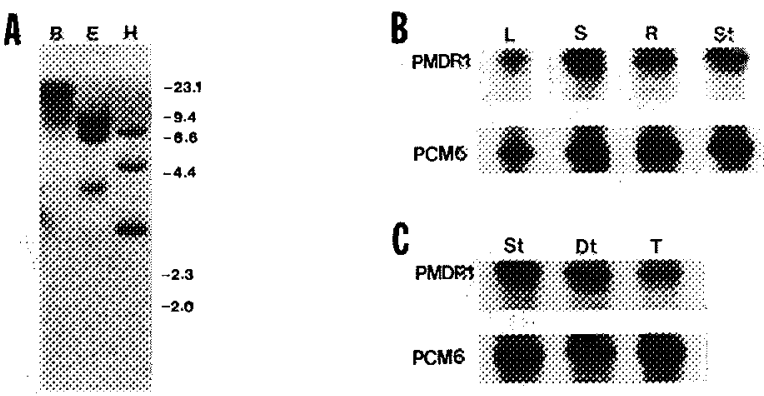

Figure 3. A. Southern analysis of PMDR 1. $10 \mu \mathrm{g}$ of potato DNA was digested with restriction enzymes and transferred onto a nylon membrane. The membrane was hybridized at $42{ }^{\circ} \mathrm{C}$ with ${ }^{32} \mathrm{P}$-labeled probe in a solution containing $50 \%$ formamide, $6 \times$ SSPE, $5 \times$ Denhardt's solution, $0.1 \% \mathrm{w} / \mathrm{v}$ SDS, and $100 \mu \mathrm{g} / \mathrm{ml}$ herring sperm DNA. The membrane was washed at $60^{\circ} \mathrm{C}$ in $0.5 \times \mathrm{SSC}$ and $0.1 \% \mathrm{w} / \mathrm{v}$ SDS. The size of the standard markers is shown in kb. B, Ban HI; E, EcoRI; H, HindIII. B. RNase protection assay showing the expression of PMDR 1 in different tissues of potato plants. $20 \mu \mathrm{g}$ of total RNA was used in each reaction. L, leaf; S, stem; R, root; St, stolon tip. The expression of potato calmodulin isoform PCM6 is shown for comparison. It has been observed that PCM6 expression is lower in leaf as compared to other organs [16]. C. RNase protection assay showing the expression of PMDR1 during the early stages of tuber development. St, stolon tip; Dt, developing tuber; T, tuber. The expression of potato calmodulin isoform (PCM6) is shown for comparison.

cating that potato has one or two MDR-like gene(s) (Fig. 3A). To study the expression of the PMDR I gene in different organs, total RNA was isolated from different organs of potato plants and developing tubers at two different stages of development [19]. The RNase protection assays were performed by using the standard protocol [13]. An SpeI/HindIII fragment of the PMDR1 coding region (nt 1711-2133, Fig. 1) was subcloned into pBluescript II KS(+) plasmid and used as a template for making the ${ }^{32} \mathrm{P}$-labeled antisense RNA probe, and the reaction mixture was analyzed on $4 \%$ polyacrylamide gel containing $7 \mathrm{M}$ urea. The ${ }^{32} \mathrm{P}-$ labeled antisense RNA probe for the potato calmodulin PCM6, which exhibits constitutive mRNA expression [16], was used for comparison. The results indicate that PMDR1 is constitutively expressed in all organs studied, with the higher expression in the stem and stolon tip (Fig. 3B). The expression was highest in the stolon tip during tuber initiation and decreased during tuber development (Fig. 3C).

A number of MDR or MDR-like genes have been isolated from different organisms [6, 14]. Although the functions of some of these genes have not been clearly identified, they are most likely to be membrane-bound transporter proteins with a wide substrate specificity. 
Most of the studies on the functions and substrates of MDR P-glycoprotein were conducted with the tumor cells, which are invariably associated with increased production of P-glycoprotein. However, several studies have consistently shown that the MDR P-glycoprotein is expressed in many normal organs, suggesting that they may play a protective role in the transport or secretion and keep toxic metabolites and xenobiotics out of these normal tissues $[2,5,6]$. Since many cytotoxic compounds transported by P-glycoproteins of mammals and other organisms are hydrophobic natural compounds derived from plants $[2,6]$, it is likely that similar transport systems may also exist in plants. Because the deduced amino acid sequence and the structural features of potato MDR-like gene (PMDR1) share a striking similarity to the mammalian P-glycoprotein, it is possible that its function is also conserved.

Calcium channel blockers and calmodulin antagonists have been known to reverse the MDR phenomenon $[5,8,17,18]$. However, the mechanism by which these agents reverse MDR effect is not fully understood [5]. Since the PMDR1 cDNA clone was isolated by screening a potato expression library using ${ }^{35} \mathrm{~S}$-labeled calmodulin, we believe that there is a direct interaction of $\mathrm{Ca}^{2+} /$ calmodulin with the PMDR 1 gene product. Recently, Schlemmer et al. [15] reported that the murine MDR3 P-glycoprotein function is downmodulated by $\mathrm{Ca}^{2+}$ /calmodulin. Their results suggest that the murine MDR3 P-glycoprotein is a calmodulinbinding protein. These results and our present study suggest that $\mathrm{Ca}^{2+} /$ calmodulin may play a regulatory role in the function of the MDR P-glycoprotein. $\mathrm{Ca}^{2+}$ and calmodulin regulate many cellular processes and growth and development in plants $[10,12]$. Balamani et al. [1] were able to block tuber induction by using $\mathrm{Ca}^{2+}$ chelators and calmodulin antagonists, suggesting a role for $\mathrm{Ca}^{2+} /$ calmodulin in tuberization. The high expression of PMDR1 mRNA in the stem and stolon tip (Fig. 3B and 3C) raises the possibility that calmodulin and its modulated proteins play a role in the tuberization process.

\section{Acknowledgements}

The support of the National Science Foundation (Grant DCB 91-4586) and National Aeronautics and Space Administration (Grant NAG-10-0061) are gratefully acknowledged.

\section{References}

1. Balamani V, Veluthambi K, Poovaiah BW: Effect of calcium on tuberization in potato (Solanum tuberosum L.). Plant Physiol 80: 856-858 (1986).

2. Broeks A, Janssen HWRM, Calafat J, Plasterk RHA: A Pglycoprotein protects Caenorhabditis elegans against natural toxins. EMBO J 14: 1858-1866 (1995).

3. Chen CJ, Clark D, Ueda K, Pastan I, Gottesman MM, Roninson IB: Genomic organization of the human multidrug resistance (MDR1) gene and origin of P-glycoproteins. J Biol Chem 265: 506-514 (1990).

4. Dudler R, Hertig C: Structure of an mdr-like gene from Arabidopsis thaliana. Evolutionary implications. J Biol Chem 267: 5882-5888 (1992)

5. Endicott JA, Ling V: The biochemistry of P-glycoproteinmediated multidrug resistance. Annu Rev Biochem 58: $137-171$ (1989).

6. Gottesman MM, Pastan I: Biochemistry of multidrug resistance mediated by the multidrug transporter. Annu Rev Biochem 62: $385-427$ (1993).

7. Higgins CF: The multidrug resistance $\mathbf{P}$-glycoprotein. Curr Opin Cell Biol 5: 684-687 (1993).

8. Kerr DJ, Graham J, Cummings J, Morrison JG, Thompson GG, Brodie MJ, Kaye SB: The effect of verapamil on the pharmacokinetics of adriamycin. Cancer Chemother Pharmacol 18: 239-242 (1986).

9. Kozak M: Point mutations define a sequence flanking the AUG initiator codon that modulates translation by eukaryotic ribosomes. Cell 44: 283-289 (1986).

10. Poovaiah BW, Reddy ASN: Calcium and signal transduction in plants. CRC Crit Rev Plant Sci 12: 185-211 (1993).

11. Reddy ASN, Takezawa D, Fromm H, Poovaiah BW: Isolation and characterization of two cDNAs that encode for calmodulinbinding proteins from corn root tips. Plant Sci 94: 109-117 (1993).

12. Roberts DM, Harmon AC: Calcium-modulated proteins: targets of intracellular calcium signals in higher plants. Annu Rev Plant Physiol Plant Mol Biol 43: 375-414 (1992).

13. Sambrook J, Fritsch EF, Maniatis T: Molecular cloning: A Laboratory Manual, 2nd ed. Cold Spring Harbor Laboratory Press, Cold Spring Harbor, NY (1989).

14. Schinkel AH, Borst P: Multidrug resistance mediated by Pglycoproteins. Semin Cancer Biol 2: 213-226 (1991).

15. Schlemmer SR, Yang CH, Sirotnak FM: Functional modulation of multidrug resistance-related P-glycoprotein by $\mathrm{Ca}^{2+}$ /calmodulin. J Biol Chem 270: 11040-11042 (1995).

16. Takezawa D, Liu ZH, An G, Poovaiah BW: Calmodulin gene family in potato: developmental and touch-induced expression of the mRNA encoding a novel isoform. Plant Mol Biol 27: 693-703 (1995).

17. Tsuruo T, Lida H, Nojiri M, Tsukagoshi, Sakurai Y: Potentiation of vincristine and adriamycin effects in human hemopoetic tumor cells lines by calcium antagonists and calmodulin inhibitors. Cancer Res 43: 2267-2272 (1983).

18. Tsuruo T, Lida H, Nojiri M, Tsukagoshi S, Sakurai Y: Circumvention of vincristine and adriamycin resistance in vitro and in vivo by calcium influx blockers. Cancer Res 43: 2905-2910 (1983).

19. Verwoerd TC, Dekker BMM, Hoekema A: A small-scale procedure for the rapid isolation of plant RNAs. Nucl Acids Res 17: 2362 (1989). 
